\title{
OLIVINE WEBSTERITE VEINS CUTTING THE RABROVO SERPENTINITES (SOUTH MACEDONIA): NEW EVIDENCE OF THE ARC SETTING OF THE EAST VARDAR OPHIOLITES?
}

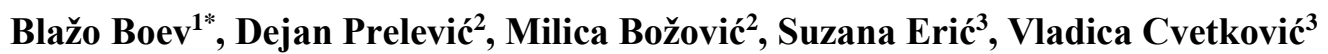 \\ ${ }^{1}$ Goce Delčev University, Štip, Republic of Macedonia \\ ${ }^{2}$ Johannes Gutenberg-Universität, Institut für Geowissenschaften, fb 09, Mineralogie, Mainz, Germany \\ ${ }^{3}$ University of Belgrade, Faculty of Mining and Geology, Belgrade, Serbia \\ *Corresponding author, e-mail: blazo.boev@ugd.edu.mk
}

\begin{abstract}
The study reports petrography and mineral chemistry data about an ultramafic vein that cuts the Rabrovo serpentinite (near Valandovo, Southern Macedonia). The serpentinite occurs as a block within a shearing zone of the East Vardar Zone tectonic mélange. The vein corresponds to medium-grained olivine websterite that sometimes displays cumulate-like textures. It is composed of low-Al orthopyroxene $\left(\mathrm{Mg \#}\left[\mathrm{mol} \mathrm{MgO} * 100 /\left(\mathrm{MgO}+\mathrm{FeO}^{\mathrm{t}}\right)\right] \sim 85\right.$, $\mathrm{Al}_{2} \mathrm{O}_{3}<2$ wt.\%), clinopyroxene $(\mathrm{Mg \#}=82-86)$, olivine $(\mathrm{Mg \#} \mathrm{84})$ and spinel $\left(\mathrm{Cr} \#\left[\mathrm{~mol} \mathrm{Cr}_{2} \mathrm{O}_{3} /\left(\mathrm{Cr}_{2} \mathrm{O}_{3}+\mathrm{Al}_{2} \mathrm{O}_{3}\right)\right] \sim 0.4\right.$, which, according to geothermometric calculations, equilibrated at $750-850{ }^{\circ} \mathrm{C}$. Its pyroxene-rich modal composition suggests that this rock cannot represent a normal lithospheric mantle. Instead, it is supposed that it was formed via magmatic precipitations in the mantle lithosphere. In addition, the Rabrovo olivine websterite shows similar mineral chemical compositions to many other orthopyroxene-rich lithologies worldwide, which origin is commonly associated with subduction settings. Therefore, its presence is considered as additional evidence that the East Vardar ophiolites represent typical supra-subduction ophiolites.
\end{abstract}

Key words: ultramafic rock; orthopyroxene; subduction; Balkan Peninsula

\section{INTRODUCTION}

The Balkan Peninsula is known from the existence of several ophiolite belts which are part of a much broader ophiolite domain that can be traced from the Western Mediterranean to the Himalaya. From the west to the east they are: Dinaridic ophiolite belt, Western Vardar ophiolites and Eastern Vardar ophiolites (e.g. [1, 2] and references therein). The geotectonic setting of all these ophiolite belts was mainly determined on the basis of the igneous members of ophiolites, mostly pillow lavas and diabase dykes, and only partly by studying depletion degrees of the underlying mantle peridotites [3-5]. Only very rare studies from this region were aimed at investigating orthopyroxene-rich lithologies [6]. On the other hand, it is very well known that ophiolites containing this lithology are commonly interpreted as originating from subductionrelated settings (e.g. [7]).
The East Vardar Zone has the most pronounced supra-subduction signature of all the Balkan ophiolite belts. This setting was first, at least indirectly, suggested by [3] Maksimović and Majer (1981) and [8] on the basis of the higher depletion extent found in the East Vardar peridotites with respect to the Dinaride and West Vardar peridotites. The most striking evidence came from a recent study of ophiolite-related volcanic rocks of the Demir Kapija ophiolites in south Macedonia [9]. Moreover, based on the composition of mantle xenoliths entrained in Palaeogene mafic alkaline rocks of East Serbia, some authors $[10,11]$ argued that the mantle underneath the present day East Serbia also possesses supra-subduction signatures. These authors further postulated that this subcontinental mantle slice may, in fact, represent suboceanic mantle portions that were accreted during the closure of the East Vardar Zone (see also [12]). One of the strong- 
est independent evidence for this hypothesis was derived from the presence and characteristics of a sub-group of olivine websterite xenoliths. [13] studied these orthopyroxene-rich xenoliths in detail and concluded that they represented lithospheric precipitates of boninite-like magmas similar to those commonly found in sub-arc settings.

In this study we report and discuss petrography and mineral chemistry of a pyroxene-rich vein cutting the serpentinite of Rabrovo (south Macedonia). The serpentinites geotectonically belong to the narrow East Vardar Zone ophiolite belt, and this is the first occurrence of orthopyroxene-rich lithology in this ophiolite zone. In a separate paper we shall present the full whole rock and mineral major and trace element geochemistry of this lithology, whereas in this study we focus on the major element compositions of main minerals. By comparing the compositions of the minerals from the Rabrovo websterite vein with those occurring in the olivine websterite xenoliths reported by [13], we shed more light on the significance of orthopyroxene-rich lithologies for determining the geotectonic setting of their host ophiolites.

\section{GEOTECTONIC SETTING}

The present day geology of Macedonia is generally interpreted as having resulted by collision between the continental margins of Adria and Eurasia [14-16]. The central parts are dominated by the Pelagonides and the Vardar Zone s.l. The Pelagonides are composed of Precambrian rocks extending northward from the Skutari-Peć line to the Drina-Ivanjica metamorphic unit [2, 17-21]. The Vardar Zone s.l. is represented by the Western and the East Vardar ophiolitic units (sensu [2]). The East Vardar ophiolites occur in the southeast part of Macedonia, where they are represented by the largest ophiolitic complex of Demir Kapija. The whole ophiolitic section is unconformably covered by the Upper Tithonian reef limestones, which are, in the northwest and the south-east covered by upper Eocene-Pliocene and Pliocene-Quaternary sediments, respectively. In the west, the East Vardar ophiolites have tectonic relationship with the Pelagonian unit, whereas the north-eastern tectonic contact with the Serbo-Macedonian Massif is characterized by a mylonite zone, and sporadically by tectonic mélange. Within this heterogeneous mélange-like zone occur numerous ophioliterelated rock associations, which are spatially detached from the main body of the Demir Kapija complex. They are represented by variably sized blocks of diabases, gabbros and serpentinites, often containing pyroxene-rich veins.

\section{EXPERIMENTAL SECTION}

Ten samples of the studied ultramafic vein were cut to produce chips for further scanning electron and microprobe investigations. The samples are first investigated petrographically using transmitted-light microscope in order to select the freshest rocks. The five freshest samples are studied on major element chemistry of the main minerals. Mineral chemistry was determined by electron microprobe (JEOL JXA 8900RL) at the Department of Geosciences, University of Mainz (Germany), using wavelength-dispersive analysis and a range of natural and synthetic standards. The data were corrected using the CITZAF procedure [22]. Detection limits were between 0.01 and $0.07 \mathrm{wt} \%$. Operating conditions were generally $15 \mathrm{kV}(20 \mathrm{kV})$ accelerating voltage, $12 \mathrm{nA}$ beam current, $1-5 \mu \mathrm{m}$ beam diameter and 15-30 s counting time on peak.

\section{RESULTS}

\section{Field occurrence and petrography of the orthopyroxene-rich veins}

The studied pyroxene-rich veins are found cutting a serpentinite block from the tectonic mélange of the East Vardar Zone. The serpentinite crops out near Rabrovo, along the road ValandovoStrumica. It appears as a $\sim 1 \mathrm{~km}$ long and a few hundreds of meters wide lens-like body that exhibits sharp tectonic contacts with the adjacent medium - to lowgrade metamorphic rocks of the Serbo-Macedonian Massif (Figure 1; [23]).

The pyroxene-rich vein represents, in fact, an approximately $1-1.5 \mathrm{~m}$ thick veining zone (Figure 2a). The veining zone consists of numerous pyroxene-rich individual veins ranging in thickness from only a few millimeters to $>15 \mathrm{~cm}$. Both host serpentinite and pyroxene-rich veins are strongly sheared and at places mylonitized and no evidence of true magmatic contacts can be observed. Therefore, the veins sometimes have typical lens-like forms or dismembered bands that resemble typical boudins.

These suggest that the presently observed contacts resulted from shearing during mylonitization and that boudin-like structures are probably formed by stretching along the shear foliation and shortening perpendicular to this. The serpentinite is composed of rare relicts of olivine and pyroxene set in a fine-grained matrix of serpentine minerals. In this stage of investigation it is not clear whether they represent remnants of mantle peridotites or those originating from the bottom of oceanic crust. 


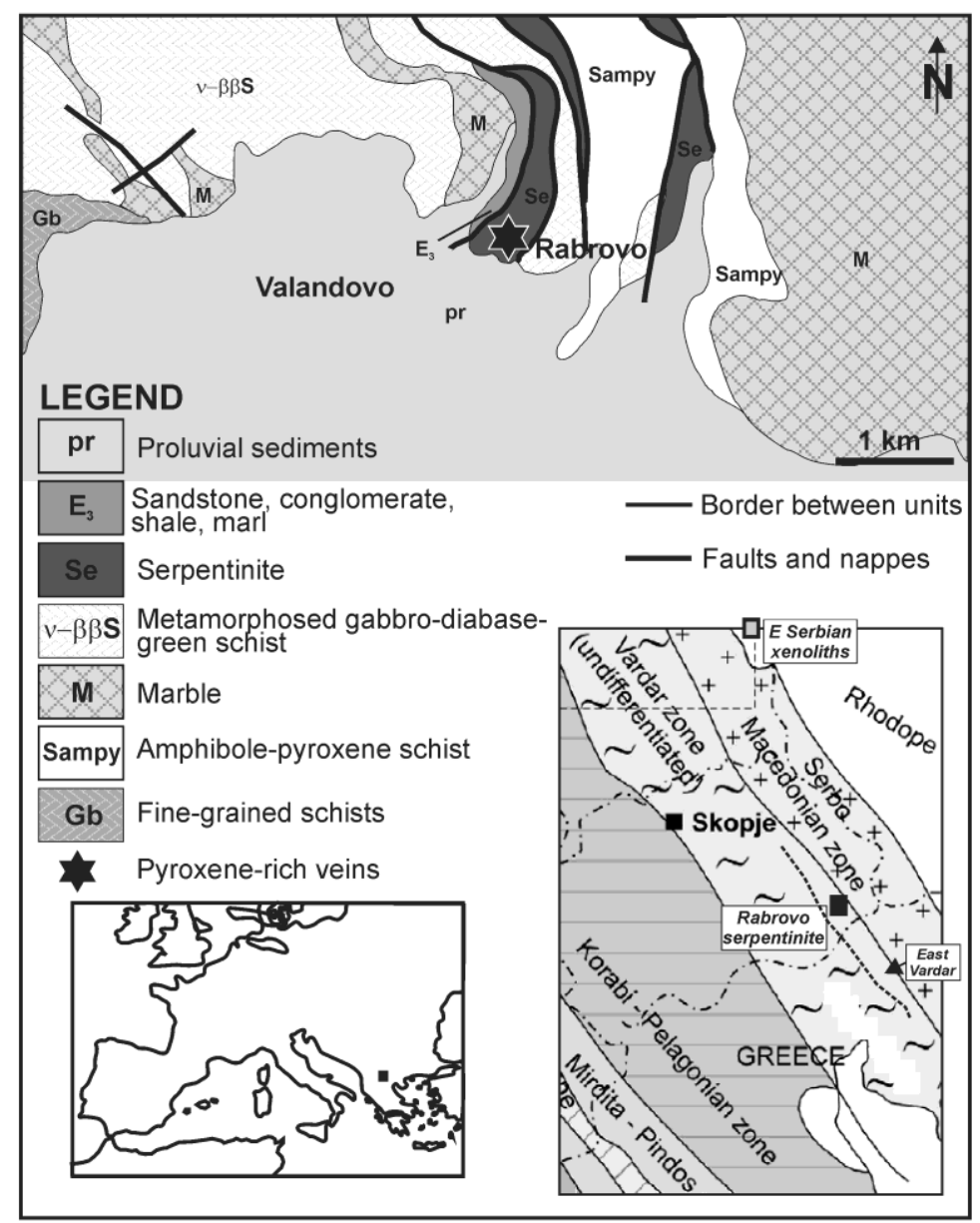

Figure 1. Geological sketch of the Rabrovo area. Compiled from the Basic Geological Map of SFR Yugoslavia 1:100000, Sheet: Gevgelija [23]; The inset is after [21]

The pyroxenite veins have medium- to finegrained granular texture. At $\mathrm{mm}$ scale the phases are merely undeformed and display sharp and mostly curvilinear contact suggesting good equilibration. It is composed of variable proportions of olivine, orthopyroxene, clinopyroxene and spinel. Apart of these predominant phases a few grains of altered amphibole are also found in some veins. The modal composition is very variable, showing considerable differences at $\mathrm{cm}$ and even $\mathrm{mm}$ scale. The differences are mostly related to different proportions of olivine and two pyroxenes. Irrespectively to the wide range in modal composition, this rock differs from normal mantle peridotites by having less than 50 $\%$ olivine, and can be classified as olivine websterite. Orthopyroxene often predominates over clinopyroxene and forms the main crystal network (Figure 2b). It is subhedral and tabular in shape and usually displays tiny exsolution lamellae (Figures 2b, d). Clinopyroxene often appears filling interstitial spaces in-between orthopyroxene crystals and giving cumulitic characteristics (Figure 2c). It sometimes forms larger pools that enclose smaller orthopyroxene grains (Figure 2d). These textural relationships can suggest that orthopyroxene crystallized shortly before and/or partly simultaneously to clinopyroxene. Olivine is, most probably, an earlier phase that crystallized before the pyroxenes. Sometimes it appears as subhedral to almost idiomorphic crystal indicating free crystallization in open space (Figure 2b). It is rather fresh with serpenttinization developed only along cracks. Spinel is represented by tiny, subhedral and mostly equidimensional to slightly elongated grains. They are isolated and enclosed by more coarse-grained silicates (Figure 2c, d).

\section{Mineral chemistry}

The studied minerals from the Rabrovo olivine websterite veins have relatively uniform major element compositions. The results of microprobe investigations are given in Tables $1-4$. The classifycation diagram of pyroxenes of Morimoto et al. [24] is given in Figure 3.

Orthopyroxene corresponds to enstatite with an average composition of $\mathrm{En}_{85} \mathrm{Fs}_{14} \mathrm{~W}_{1}$. 

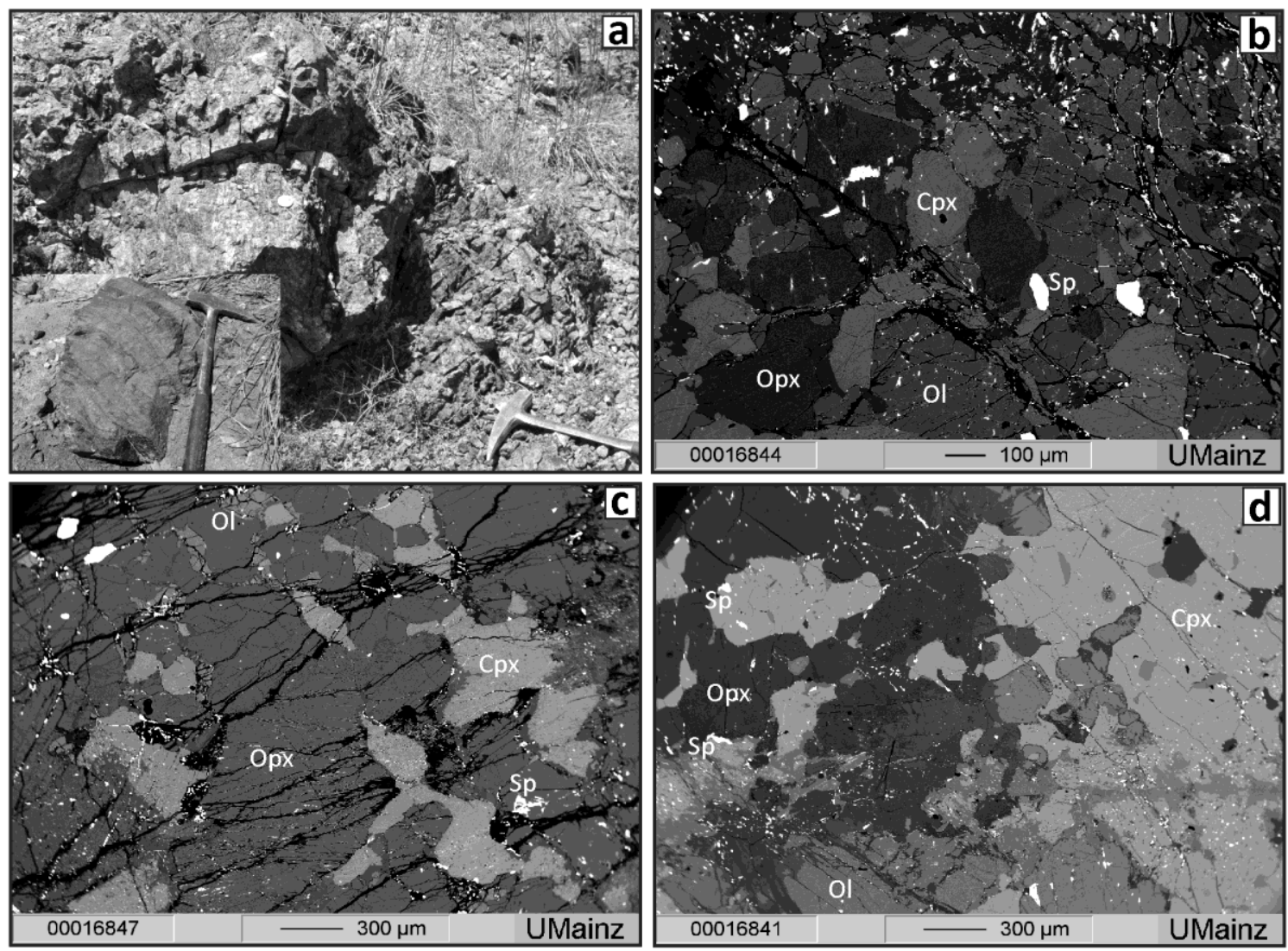

Figure 2. Field photos and BSE images of the studied olivine websterite. a - Field outcrop of the Rabrovo olivine websterite; the inset displays cm-thick interfingering of serpentinite and olivine websterite rocks; $b$ - granular texture with subhedral to almost euhedral orthopyroxene and olivine and subordinate anhedral clinopyroxene; $\mathrm{c}-\mathrm{a}$ cumulate-

like texture with interstitial clinopyroxene surrounded by orthopyroxene; $d-a$ larger clinopyroxene pool in the olivine websterite (right-hand side of the photo)

It is characterized by uniform $\mathrm{Mg \#} \mathrm{[mol}$ $\left.\mathrm{MgO}^{*} 100 /\left(\mathrm{MgO}+\mathrm{FeO}^{t}\right)\right]$ values mostly ranging between 84 and $86 . \mathrm{Al}_{2} \mathrm{O}_{3}$ and $\mathrm{CaO}$ contents are relatively low and range from 1 to $1.5 \mathrm{wt} . \%$, and from 0.3 to $0.6 \mathrm{wt} . \%$, respectively. Chromium and nickel contents vary between $0.15 \%$ wt. $\%$ and 0.4 wt.\% and $\sim 0.5$ wt. $\% \mathrm{Cr}_{2} \mathrm{O}_{3}$ and $\mathrm{NiO}$, respectively. $\mathrm{Al}_{2} \mathrm{O}_{3}, \mathrm{CaO}$ and $\mathrm{Cr}_{2} \mathrm{O}_{3}$ contents in this orthopyroxene are comparable from those found in orthopyroxene from the East Vardar peridotites, and are remarkably lower than those displayed by orthopyroxene from peridotite of Dinaride and West Vardar ophiolites [25, 26]. In comparison to orthopyroxene from East Serbian mantle xenoliths, there is a clear compositional similarity with orthopyroxene from spinel-poor olivine websterite xenoliths (Figure 4). The origin of these olivine websterite xenoliths is interpreted in terms of lithospheric crystallization of high-Si-Mg boninite-like magmas [11].

Clinopyroxene shows narrow compositional transitions from diopside to augite with an average formula of $\mathrm{En}_{\sim 46} \mathrm{Fs}_{\sim 8} \mathrm{~W}_{\sim 46}$. It is also characterized by relatively uniform Mg\#s mostly between 85 and 86 . Elements that commonly reflects the fertility of the magmatic source are present in very low concentrations, for instance: $\mathrm{Al}_{2} \mathrm{O}_{3}=1-1.6 \mathrm{wt} . \%$, $\mathrm{TiO}_{2}=0.04-0.06$ wt. $\%$ and $\mathrm{Na}_{2} \mathrm{O}<0.1$ wt. $\%$. Chromium contents are moderate and range between $0.3-0.4$ wt. $\% \mathrm{Cr}_{2} \mathrm{O}_{3}$. In Figure 5 is given $\mathrm{Mg \#}$ vs $\mathrm{Al}_{2} \mathrm{O}_{3}$ (wt.\%) plot for clinopyroxene. It is evident that the clinopyroxene that occurs in the studied olivine websterite veins is compositionally very similar to the clinopyroxene from olivine websterite mantle xenoliths derived from the East Serbian subcontinental mantle $[11,13]$.

Olivine is relatively uniform and corresponds to Fo83-85. It is characterized by low calcium contents $(\mathrm{CaO}=0.02-0.08$ wt. \% $)$ and rather uniform nickel concentrations ranging $0.24-0.28$ wt. $\% \mathrm{NiO}$.

Spinel is chromium rich with $\mathrm{Cr} \#[\mathrm{molCr} 2 \mathrm{O} 3 /(\mathrm{Cr} 2 \mathrm{O} 3+\mathrm{Al} 2 \mathrm{O} 3)]$ ranging between 0.38 and 0.45 and $\mathrm{Fe} \#[\mathrm{~mol} \mathrm{FeOt} /(\mathrm{MgO}+\mathrm{FeOt})]$ values about 0.5 . Average olivine-spinel compositions plot off the "mantle array" ([27]; not shown), because their olivine shows lower Fo contents for the given high $\mathrm{Cr} \#$ in spinel. The same is observed for the East Serbian olivine websterite xenoliths $[10,11]$, although spinels from the xenoliths have higher Cr\#s (> 0.65). 
Table 1. Microprobe analyses of orthopyroxene from olivine websterite of Rabrovo

\begin{tabular}{|c|c|c|c|c|c|c|c|c|c|c|c|c|c|c|c|c|c|c|c|c|}
\hline No. & 10 & 12 & 13 & 14 & 15 & 16 & 19 & 20 & 21 & 22 & 23 & 24 & 25 & 26 & 27 & 59 & 61 & 63 & 65 & 94 \\
\hline & 55.6 & 56.8 & 56.9 & 56.5 & 56.4 & 56.3 & 55.8 & 56.0 & 56.3 & 56.0 & 55.8 & 55.8 & 55.2 & 55.3 & 55.7 & 53.3 & 53.4 & 53.4 & 53.4 & 55.2 \\
\hline $\mathrm{SiO}_{2}$ & 6 & 0 & 0 & 4 & 8 & 6 & 4 & 2 & 2 & 0 & 1 & 5 & 2 & 6 & 2 & 2 & 8 & 2 & 9 & 7 \\
\hline $\mathrm{TiO}_{2}$ & 0.02 & 0.02 & 0.03 & 0.04 & 0.03 & 0.02 & 0.03 & 0.03 & 0.03 & 0.03 & 0.02 & 0.03 & 0.04 & 0.03 & 0.04 & 0.03 & 0.01 & 0.03 & 0.03 & 0.02 \\
\hline $\mathrm{Al}_{2} \mathrm{O}_{3}$ & 1.39 & 1.35 & 1.37 & 1.35 & 1.37 & 1.35 & 1.43 & 1.41 & 1.38 & 1.36 & 1.35 & 1.35 & 1.33 & 1.34 & 1.31 & 1.29 & 1.31 & 1.35 & 1.39 & 1.38 \\
\hline $\mathrm{FeO}$ & 9.19 & 8.97 & 9.13 & 9.11 & 9.10 & 9.28 & 9.08 & 9.08 & 9.09 & 9.24 & 9.21 & 9.10 & 9.09 & 9.08 & 9.12 & 9.29 & 9.22 & 9.35 & 9.19 & 9.54 \\
\hline \multirow[t]{2}{*}{ MnO } & 0.27 & 0.26 & 0.25 & 0.25 & 0.25 & 0.25 & 0.25 & 0.25 & 0.27 & 0.23 & 0.25 & 0.26 & 0.25 & 0.24 & 0.24 & 0.25 & 0.24 & 0.26 & 0.26 & 0.27 \\
\hline & 32.4 & 32.2 & 32.1 & 31.7 & 31.9 & 31.9 & 31.9 & 31.8 & 31.9 & 31.8 & 31.9 & 32.0 & 31.7 & 32.0 & 31.9 & 32.0 & 32.0 & 32.1 & 31.8 & 31.9 \\
\hline MgO & 6 & 2 & 1 & 9 & 0 & 1 & 9 & 7 & 9 & 7 & 5 & 0 & 1 & 6 & 2 & 3 & 1 & 0 & 4 & 4 \\
\hline $\mathrm{CaO}$ & 0.58 & 0.54 & 0.55 & 0.56 & 0.58 & 0.55 & 0.64 & 0.65 & 0.59 & 0.66 & 0.65 & 0.65 & 0.63 & 0.63 & 0.61 & 0.59 & 0.58 & 0.54 & 0.54 & 0.48 \\
\hline $\mathrm{Cr}_{2} \mathrm{O}_{3}$ & 0.37 & 0.35 & 0.34 & 0.35 & 0.36 & 0.34 & 0.32 & 0.32 & 0.31 & 0.32 & 0.29 & 0.31 & 0.30 & 0.30 & 0.30 & 0.25 & 0.26 & 0.26 & 0.26 & 0.26 \\
\hline \multirow[t]{2}{*}{$\mathrm{NiO}$} & 0.04 & 0.06 & 0.05 & 0.05 & 0.04 & 0.06 & 0.05 & 0.05 & 0.05 & 0.06 & 0.07 & 0.05 & 0.07 & 0.05 & 0.05 & 0.05 & 0.05 & 0.04 & 0.03 & 0.05 \\
\hline & 99.9 & 100. & 100. & 100. & 100. & 100. & 99.6 & 99.6 & 100. & 99.7 & 99.6 & 99.6 & 98.6 & 99.0 & 99.3 & 97.1 & 97.1 & 97.3 & 97.0 & 99.2 \\
\hline Total & 7 & 57 & 72 & 02 & 11 & 11 & 3 & 8 & 04 & 6 & 0 & 0 & 4 & 9 & 1 & 0 & 5 & 5 & 3 & 0 \\
\hline \multicolumn{21}{|c|}{$\begin{array}{l}\text { Site allocations (6 } \\
\text { O) }\end{array}$} \\
\hline & 1.94 & 1.97 & 1.97 & 1.97 & 1.97 & 1.96 & 1.95 & 1.96 & 1.96 & 1.96 & 1.95 & 1.96 & 1.95 & 1.95 & 1.96 & 1.91 & 1.91 & 1.91 & 1.92 & 1.94 \\
\hline \multirow[t]{2}{*}{$\mathbf{S i}$} & 4 & 2 & 3 & 5 & 2 & 9 & 9 & 5 & 8 & 4 & 9 & 0 & 7 & 1 & 1 & 4 & 8 & 2 & 2 & 8 \\
\hline & 0.05 & 0.02 & 0.02 & 0.02 & 0.02 & 0.03 & 0.04 & 0.03 & 0.03 & 0.03 & 0.04 & 0.04 & 0.04 & 0.04 & 0.03 & 0.05 & 0.05 & 0.05 & 0.05 & 0.05 \\
\hline \multirow[t]{2}{*}{ Al } & 6 & 8 & 7 & 5 & 8 & 1 & 1 & 5 & 2 & 6 & 1 & 0 & 3 & 9 & 9 & 4 & 5 & 7 & 9 & 2 \\
\hline & 0.00 & 0.00 & 0.00 & 0.00 & 0.00 & 0.00 & 0.00 & 0.00 & 0.00 & 0.00 & 0.00 & 0.00 & 0.00 & 0.00 & 0.00 & 0.03 & 0.02 & 0.03 & 0.01 & 0.00 \\
\hline \multirow[t]{2}{*}{$\mathrm{Fe}^{3}$} & 0 & 0 & 0 & 0 & 0 & 0 & 0 & 0 & 0 & 0 & 0 & 0 & 0 & 0 & 0 & 2 & 6 & 1 & 9 & 0 \\
\hline & 2.00 & 2.00 & 2.00 & 2.00 & 2.00 & 2.00 & 2.00 & 2.00 & 2.00 & 2.00 & 2.00 & 2.00 & 2.00 & 2.00 & 2.00 & 2.00 & 2.00 & 2.00 & 2.00 & 2.00 \\
\hline \multirow[t]{2}{*}{$T$} & 0 & 0 & 0 & 0 & 0 & 0 & 0 & 0 & 0 & 0 & 0 & 0 & 0 & 0 & 0 & 0 & 0 & 0 & 0 & 0 \\
\hline & 0.00 & 0.02 & 0.02 & 0.03 & 0.02 & 0.02 & 0.01 & 0.02 & 0.02 & 0.02 & 0.01 & 0.01 & 0.01 & 0.00 & 0.01 & 0.00 & 0.00 & 0.00 & 0.00 & 0.00 \\
\hline Al & 1 & 7 & 9 & 0 & 8 & 5 & 8 & 4 & 5 & 0 & 5 & 6 & 3 & 7 & 6 & 0 & 0 & 0 & 0 & 5 \\
\hline & 0.04 & 0.00 & 0.00 & 0.00 & 0.00 & 0.00 & 0.01 & 0.00 & 0.00 & 0.00 & 0.01 & 0.01 & 0.02 & 0.03 & 0.01 & 0.07 & 0.07 & 0.07 & 0.06 & 0.03 \\
\hline $\mathrm{Fe}^{3}$ & 5 & 0 & 0 & 0 & 0 & 0 & 3 & 0 & 0 & 6 & 7 & 4 & 0 & 3 & 3 & 8 & 4 & 9 & 9 & 8 \\
\hline & 0.00 & 0.00 & 0.00 & 0.00 & 0.00 & 0.00 & 0.00 & 0.00 & 0.00 & 0.00 & 0.00 & 0.00 & 0.00 & 0.00 & 0.00 & 0.00 & 0.00 & 0.00 & 0.00 & 0.00 \\
\hline $\mathrm{Ti}$ & 1 & 1 & 1 & 1 & 1 & 0 & 1 & 1 & 1 & 1 & 0 & 1 & 1 & 1 & 1 & 1 & 0 & 1 & 1 & 1 \\
\hline & 0.01 & 0.01 & 0.00 & 0.01 & 0.01 & 0.01 & 0.00 & 0.00 & 0.00 & 0.00 & 0.00 & 0.00 & 0.00 & 0.00 & 0.00 & 0.00 & 0.00 & 0.00 & 0.00 & 0.00 \\
\hline $\mathrm{Cr}$ & 0 & 0 & 9 & 0 & 0 & 0 & 9 & 9 & 9 & 9 & 8 & 9 & 8 & 8 & 8 & 7 & 7 & 7 & 7 & 7 \\
\hline & 0.00 & 0.00 & 0.00 & 0.00 & 0.00 & 0.00 & 0.00 & 0.00 & 0.00 & 0.00 & 0.00 & 0.00 & 0.00 & 0.00 & 0.00 & 0.00 & 0.00 & 0.00 & 0.00 & 0.00 \\
\hline $\mathrm{Ni}$ & 1 & 2 & 1 & 1 & 1 & 2 & 1 & 1 & 2 & 2 & 2 & 1 & 2 & 1 & 1 & 1 & 1 & 1 & 1 & 1 \\
\hline & 0.94 & 0.96 & 0.95 & 0.95 & 0.96 & 0.96 & 0.95 & 0.96 & 0.96 & 0.96 & 0.95 & 0.95 & 0.95 & 0.95 & 0.96 & 0.91 & 0.91 & 0.91 & 0.92 & 0.94 \\
\hline Mg & 3 & 1 & 9 & 8 & 0 & 4 & 8 & 5 & 4 & 3 & 8 & 9 & 6 & 0 & 1 & 3 & 7 & 2 & 2 & 7 \\
\hline & 0.00 & 0.00 & 0.00 & 0.00 & 0.00 & 0.00 & 0.00 & 0.00 & 0.00 & 0.00 & 0.00 & 0.00 & 0.00 & 0.00 & 0.00 & 0.00 & 0.00 & 0.00 & 0.00 & 0.00 \\
\hline $\mathrm{Fe}^{2}$ & 0 & 0 & 0 & 0 & 0 & 0 & 0 & 0 & 0 & 0 & 0 & 0 & 0 & 0 & 0 & 0 & 0 & 0 & 0 & 0 \\
\hline & 0.00 & 0.00 & 0.00 & 0.00 & 0.00 & 0.00 & 0.00 & 0.00 & 0.00 & 0.00 & 0.00 & 0.00 & 0.00 & 0.00 & 0.00 & 0.00 & 0.00 & 0.00 & 0.00 & 0.00 \\
\hline Mn & 0 & 0 & 0 & 0 & 0 & 0 & 0 & 0 & 0 & 0 & 0 & 0 & 0 & 0 & 0 & 0 & 0 & 0 & 0 & 0 \\
\hline & 1.00 & 1.00 & 1.00 & 1.00 & 1.00 & 1.00 & 1.00 & 1.00 & 1.00 & 1.00 & 1.00 & 1.00 & 1.00 & 1.00 & 1.00 & 1.00 & 1.00 & 1.00 & 1.00 & 1.00 \\
\hline M1 & 0 & 0 & 0 & 0 & 0 & 0 & 0 & 0 & 0 & 0 & 0 & 0 & 0 & 0 & 0 & 0 & 0 & 0 & 0 & 0 \\
\hline & 0.74 & 0.70 & 0.70 & 0.69 & 0.70 & 0.69 & 0.71 & 0.70 & 0.70 & 0.70 & 0.71 & 0.71 & 0.71 & 0.73 & 0.71 & 0.80 & 0.79 & 0.80 & 0.78 & 0.73 \\
\hline Mg & 7 & 7 & 1 & 8 & 0 & 8 & 5 & 2 & 3 & 3 & 4 & 5 & 9 & 4 & 4 & 1 & 5 & 1 & 4 & 1 \\
\hline & 0.22 & 0.26 & 0.26 & 0.26 & 0.26 & 0.27 & 0.25 & 0.26 & 0.26 & 0.26 & 0.25 & 0.25 & 0.24 & 0.23 & 0.25 & 0.16 & 0.17 & 0.17 & 0.18 & 0.24 \\
\hline $\mathrm{Fe}^{2}$ & 4 & 0 & 5 & 6 & 6 & 1 & 4 & 6 & 6 & 5 & 4 & 3 & 9 & 5 & 6 & 9 & 6 & 0 & 8 & 3 \\
\hline & 0.00 & 0.00 & 0.00 & 0.00 & 0.00 & 0.00 & 0.00 & 0.00 & 0.00 & 0.00 & 0.00 & 0.00 & 0.00 & 0.00 & 0.00 & 0.00 & 0.00 & 0.00 & 0.00 & 0.00 \\
\hline Mn & 8 & 8 & 7 & 7 & 7 & 7 & 8 & 7 & 8 & 7 & 8 & 8 & 8 & 7 & 7 & 8 & 7 & 8 & 8 & 8 \\
\hline & 0.02 & 0.02 & 0.02 & 0.02 & 0.02 & 0.02 & 0.02 & 0.02 & 0.02 & 0.02 & 0.02 & 0.02 & 0.02 & 0.02 & 0.02 & 0.02 & 0.02 & 0.02 & 0.02 & 0.01 \\
\hline $\mathrm{Ca}$ & 2 & 0 & 0 & 1 & 2 & 1 & 4 & 4 & 2 & 5 & 4 & 4 & 4 & 4 & 3 & 3 & 2 & 1 & 1 & 8 \\
\hline & 0.00 & 0.00 & 0.00 & 0.00 & 0.00 & 0.00 & 0.00 & 0.00 & 0.00 & 0.00 & 0.00 & 0.00 & 0.00 & 0.00 & 0.00 & 0.00 & 0.00 & 0.00 & 0.00 & 0.00 \\
\hline $\mathrm{Na}$ & 0 & 0 & 0 & 0 & 0 & 0 & 0 & 0 & 0 & 0 & 0 & 0 & 0 & 0 & 0 & 0 & 0 & 0 & 0 & 0 \\
\hline & 0.00 & 0.00 & 0.00 & 0.00 & 0.00 & 0.00 & 0.00 & 0.00 & 0.00 & 0.00 & 0.00 & 0.00 & 0.00 & 0.00 & 0.00 & 0.00 & 0.00 & 0.00 & 0.00 & 0.00 \\
\hline $\mathbf{K}$ & 0 & 0 & 0 & 0 & 0 & 0 & 0 & 0 & 0 & 0 & 0 & 0 & 0 & 0 & 0 & 0 & 0 & 0 & 0 & 0 \\
\hline & 1.00 & 0.99 & 0.99 & 0.99 & 0.99 & 0.99 & 1.00 & 1.00 & 0.99 & 1.00 & 1.00 & 1.00 & 1.00 & 1.00 & 1.00 & 1.00 & 1.00 & 1.00 & 1.00 & 1.00 \\
\hline M2 & 0 & 5 & 3 & 2 & 4 & 8 & 0 & 0 & 8 & 0 & 0 & 0 & 0 & 0 & 0 & 0 & 0 & 0 & 0 & 0 \\
\hline & 85.0 & 85.2 & 85.0 & 84.9 & 84.9 & 84.7 & 84.8 & 84.8 & 84.9 & 84.6 & 84.6 & 84.8 & 84.7 & 84.9 & 84.8 & 84.7 & 84.8 & 84.7 & 84.8 & 84.5 \\
\hline En & 1 & 7 & 2 & 1 & 2 & 4 & 9 & 2 & 3 & 3 & 9 & 4 & 8 & 4 & 7 & 1 & 3 & 4 & 4 & 3 \\
\hline & 13.9 & 13.7 & 13.9 & 14.0 & 13.9 & 14.2 & 13.9 & 13.9 & 13.9 & 14.1 & 14.0 & 13.9 & 14.0 & 13.8 & 13.9 & 14.1 & 14.0 & 14.2 & 14.1 & 14.5 \\
\hline Fs & 0 & 1 & 4 & 2 & 6 & 0 & 0 & 4 & 4 & 1 & 8 & 3 & 2 & 6 & 6 & 6 & 6 & 3 & 3 & 7 \\
\hline Wo & 1.09 & 1.02 & 1.04 & 1.07 & 1.12 & 1.05 & 1.22 & 1.24 & 1.13 & 1.26 & 1.24 & 1.23 & 1.21 & 1.20 & 1.16 & 1.13 & 1.10 & 1.03 & 1.03 & 0.91 \\
\hline & 85.9 & 86.1 & 85.9 & 85.8 & 85.8 & 85.6 & 85.9 & 85.8 & 85.9 & 85.7 & 85.7 & 85.9 & 85.8 & 85.9 & 85.8 & 85.6 & 85.7 & 85.6 & 85.7 & 85.3 \\
\hline Mg\# & 5 & 5 & 1 & 3 & 8 & 4 & 3 & 9 & 0 & 1 & 5 & 0 & 1 & 7 & 7 & 8 & 8 & 2 & 2 & 0 \\
\hline
\end{tabular}


Table 2. Microprobe analyses of clinopyroxene from olivine websterite of Rabrovo (in \%)

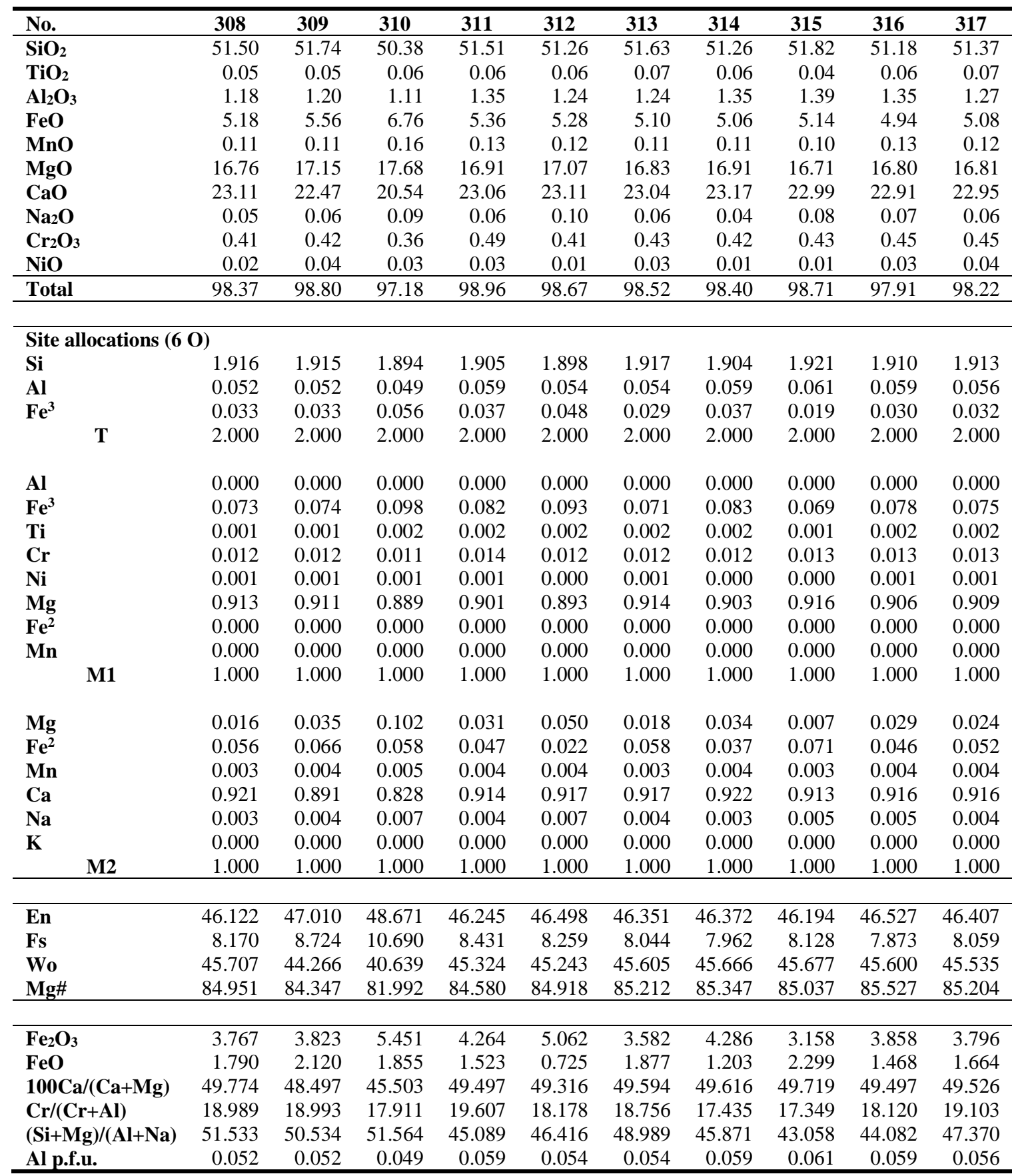


Table 3. Microprobe analyses of olivine from olivine websterite of Rabrovo (in \%)

\begin{tabular}{|c|c|c|c|c|c|c|c|c|c|c|c|c|c|}
\hline No. & 49 & 51 & 53 & 55 & 57 & 67 & 68 & 80 & 82 & 84 & 89 & 90 & 91 \\
\hline $\mathrm{SiO}_{2}$ & 39.12 & 39.31 & 39.16 & 38.77 & 38.68 & 39.30 & 38.94 & 39.06 & 38.96 & 39.26 & 39.79 & 39.32 & 39.53 \\
\hline $\mathrm{FeO}$ & 14.65 & 14.56 & 14.21 & 14.50 & 14.44 & 14.27 & 14.21 & 15.43 & 15.60 & 15.18 & 14.70 & 14.64 & 14.62 \\
\hline MnO & 0.25 & 0.25 & 0.22 & 0.21 & 0.22 & 0.23 & 0.25 & 0.27 & 0.26 & 0.26 & 0.25 & 0.24 & 0.23 \\
\hline MgO & 45.90 & 45.79 & 45.99 & 45.78 & 45.25 & 45.83 & 45.68 & 44.84 & 44.86 & 44.90 & 45.99 & 45.79 & 45.68 \\
\hline $\mathrm{CaO}$ & 0.02 & 0.02 & 0.01 & 0.04 & 0.08 & 0.06 & 0.09 & 0.07 & 0.05 & 0.06 & 0.02 & 0.02 & 0.03 \\
\hline $\mathrm{Cr}_{2} \mathrm{O}_{3}$ & 0.03 & 0.02 & 0.03 & 0.04 & 0.02 & 0.05 & 0.04 & 0.03 & 0.04 & 0.03 & 0.02 & 0.01 & 0.03 \\
\hline $\mathrm{NiO}$ & 0.24 & 0.23 & 0.25 & 0.24 & 0.24 & 0.24 & 0.22 & 0.21 & 0.22 & 0.22 & 0.25 & 0.25 & 0.25 \\
\hline Total & 100.20 & 100.17 & 99.87 & 99.57 & 98.94 & 99.98 & 99.43 & 99.91 & 99.99 & 99.91 & 101.01 & 100.28 & 100.37 \\
\hline \multicolumn{14}{|c|}{ Site allocations (4 O) } \\
\hline Si & 0.982 & 0.986 & 0.984 & 0.979 & 0.983 & 0.986 & 0.983 & 0.987 & 0.984 & 0.990 & 0.989 & 0.986 & 0.989 \\
\hline $\mathbf{Z}$ & 0.982 & 0.986 & 0.984 & 0.979 & 0.983 & 0.986 & 0.983 & 0.987 & 0.984 & 0.990 & 0.989 & 0.986 & 0.989 \\
\hline $\mathbf{T i}$ & 0.000 & 0.000 & 0.000 & 0.000 & 0.000 & 0.000 & 0.000 & 0.000 & 0.000 & 0.000 & 0.000 & 0.000 & 0.000 \\
\hline Al & 0.000 & 0.000 & 0.000 & 0.000 & 0.000 & 0.000 & 0.000 & 0.000 & 0.000 & 0.000 & 0.000 & 0.000 & 0.000 \\
\hline $\mathrm{Fe}^{2}$ & 0.307 & 0.305 & 0.299 & 0.306 & 0.307 & 0.300 & 0.300 & 0.326 & 0.330 & 0.320 & 0.306 & 0.307 & 0.306 \\
\hline Mn & 0.005 & 0.005 & 0.005 & 0.004 & 0.005 & 0.005 & 0.005 & 0.006 & 0.005 & 0.006 & 0.005 & 0.005 & 0.005 \\
\hline Mg & 1.717 & 1.712 & 1.723 & 1.724 & 1.714 & 1.715 & 1.720 & 1.688 & 1.689 & 1.688 & 1.705 & 1.711 & 1.704 \\
\hline $\mathbf{C a}$ & 0.001 & 0.001 & 0.000 & 0.001 & 0.002 & 0.002 & 0.002 & 0.002 & 0.001 & 0.002 & 0.000 & 0.001 & 0.001 \\
\hline $\mathbf{N a}$ & 0.000 & 0.000 & 0.000 & 0.000 & 0.000 & 0.000 & 0.000 & 0.000 & 0.000 & 0.000 & 0.000 & 0.000 & 0.000 \\
\hline $\mathbf{K}$ & 0.000 & 0.000 & 0.000 & 0.000 & 0.000 & 0.000 & 0.000 & 0.000 & 0.000 & 0.000 & 0.000 & 0.000 & 0.000 \\
\hline $\mathrm{Cr}$ & 0.000 & 0.000 & 0.001 & 0.001 & 0.000 & 0.001 & 0.001 & 0.001 & 0.001 & 0.001 & 0.000 & 0.000 & 0.001 \\
\hline $\mathrm{Ni}$ & 0.005 & 0.005 & 0.005 & 0.005 & 0.005 & 0.005 & 0.005 & 0.004 & 0.004 & 0.004 & 0.005 & 0.005 & 0.005 \\
\hline $\mathbf{X}$ & 2.036 & 2.028 & 2.032 & 2.041 & 2.034 & 2.027 & 2.033 & 2.027 & 2.031 & 2.020 & 2.021 & 2.029 & 2.021 \\
\hline Mg\# & 84.81 & 84.86 & 85.23 & 84.91 & 84.82 & 85.13 & 85.14 & 83.82 & 83.68 & 84.06 & 84.80 & 84.79 & 84.78 \\
\hline Fo & 84.60 & 84.64 & 85.03 & 84.73 & 84.62 & 84.92 & 84.92 & 83.58 & 83.45 & 83.82 & 84.58 & 84.58 & 84.57 \\
\hline $\mathbf{F a}$ & 15.15 & 15.10 & 14.74 & 15.05 & 15.15 & 14.83 & 14.82 & 16.13 & 16.28 & 15.90 & 15.17 & 15.17 & 15.18 \\
\hline
\end{tabular}

Table 4. Microprobe analyses of spinels from olivine websterite of Rabrovo (in \%)

\begin{tabular}{|c|c|c|c|c|c|c|c|c|c|c|c|c|c|}
\hline No. & 45 & 46 & 47 & 48 & 73 & 75 & 77 & 78 & 122 & 123 & 124 & 125 & 126 \\
\hline $\mathrm{SiO}_{2}$ & 0.02 & 0.06 & 0.03 & 0.08 & 0.09 & 0.06 & 0.05 & 5.91 & 0.93 & 0.06 & 0.02 & 0.02 & 0.04 \\
\hline $\mathrm{TiO}_{2}$ & 0.11 & 0.11 & 0.11 & 0.10 & 0.07 & 0.08 & 0.07 & 0.07 & 0.16 & 0.16 & 0.15 & 0.17 & 0.16 \\
\hline $\mathrm{V}_{2} \mathrm{O}_{3}$ & 0.40 & 0.39 & 0.38 & 0.40 & 0.37 & 0.35 & 0.40 & 0.27 & 0.37 & 0.33 & 0.37 & 0.37 & 0.38 \\
\hline $\mathrm{Al}_{2} \mathrm{O}_{3}$ & 29.57 & 29.25 & 29.63 & 30.83 & 33.21 & 33.46 & 33.62 & 25.41 & 32.25 & 34.36 & 34.26 & 34.54 & 33.60 \\
\hline $\mathrm{Cr}_{2} \mathrm{O}_{3}$ & 36.59 & 36.89 & 36.85 & 34.85 & 32.31 & 32.47 & 32.14 & 25.59 & 32.09 & 31.77 & 31.95 & 31.76 & 32.11 \\
\hline FeOt & 22.83 & 23.81 & 22.91 & 22.91 & 23.83 & 23.30 & 23.13 & 27.24 & 23.00 & 22.30 & 22.59 & 22.60 & 22.86 \\
\hline MnO & 0.26 & 0.32 & 0.27 & 0.27 & 0.27 & 0.26 & 0.25 & 0.48 & 0.23 & 0.21 & 0.22 & 0.22 & 0.23 \\
\hline MgO & 10.14 & 9.66 & 10.09 & 10.29 & 10.06 & 10.40 & 10.54 & 9.79 & 11.01 & 11.19 & 11.07 & 11.08 & 10.89 \\
\hline $\mathrm{CaO}$ & 0.08 & 0.04 & 0.04 & 0.03 & 0.10 & 0.12 & 0.19 & 2.08 & 0.02 & 0.01 & 0.01 & 0.00 & 0.01 \\
\hline $\mathrm{NiO}$ & 0.08 & 0.07 & 0.06 & 0.06 & 0.07 & 0.06 & 0.08 & 0.05 & 0.10 & 0.12 & 0.12 & 0.13 & 0.11 \\
\hline Total & 100.08 & 100.60 & 100.38 & 99.83 & 100.38 & 100.56 & 100.48 & 96.89 & 100.15 & 100.51 & 100.76 & 100.90 & 100.39 \\
\hline $\mathrm{Cr}$ & 0.889 & 0.897 & 0.892 & 0.844 & 0.774 & 0.773 & 0.765 & 0.740 & 0.779 & 0.750 & 0.754 & 0.747 & 0.764 \\
\hline $\mathbf{T i}$ & 0.003 & 0.002 & 0.003 & 0.002 & 0.002 & 0.002 & 0.002 & 0.002 & 0.004 & 0.004 & 0.003 & 0.004 & 0.004 \\
\hline $\mathbf{V}$ & 0.008 & 0.008 & 0.008 & 0.008 & 0.007 & 0.007 & 0.008 & 0.007 & 0.007 & 0.007 & 0.007 & 0.007 & 0.007 \\
\hline Al & 1.071 & 1.061 & 1.070 & 1.114 & 1.186 & 1.189 & 1.194 & 1.096 & 1.167 & 1.210 & 1.205 & 1.212 & 1.192 \\
\hline $\mathrm{Fe}^{3+}$ & 0.018 & 0.020 & 0.016 & 0.020 & 0.021 & 0.019 & 0.021 & 0.146 & 0.031 & 0.019 & 0.019 & 0.018 & 0.021 \\
\hline $\mathrm{Fe}^{2+}$ & 0.533 & 0.554 & 0.538 & 0.529 & 0.541 & 0.529 & 0.521 & 0.395 & 0.496 & 0.501 & 0.508 & 0.509 & 0.512 \\
\hline Mn & 0.007 & 0.008 & 0.007 & 0.007 & 0.007 & 0.007 & 0.006 & 0.015 & 0.006 & 0.005 & 0.006 & 0.006 & 0.006 \\
\hline Mg & 0.464 & 0.443 & 0.461 & 0.470 & 0.454 & 0.467 & 0.473 & 0.534 & 0.504 & 0.498 & 0.492 & 0.491 & 0.488 \\
\hline Co & 0.002 & 0.001 & 0.001 & 0.001 & 0.002 & 0.003 & 0.005 & 0.061 & 0.001 & 0.000 & 0.000 & 0.000 & 0.000 \\
\hline $\mathrm{Ni}$ & 0.002 & 0.002 & 0.002 & 0.002 & 0.002 & 0.002 & 0.002 & 0.002 & 0.003 & 0.003 & 0.003 & 0.003 & 0.003 \\
\hline Mg\# & 0.47 & 0.44 & 0.46 & 0.47 & 0.46 & 0.47 & 0.48 & 0.57 & 0.50 & 0.50 & 0.49 & 0.49 & 0.49 \\
\hline Cr\# & 0.45 & 0.46 & 0.45 & 0.43 & 0.39 & 0.39 & 0.39 & 0.40 & 0.40 & 0.38 & 0.38 & 0.38 & 0.39 \\
\hline
\end{tabular}




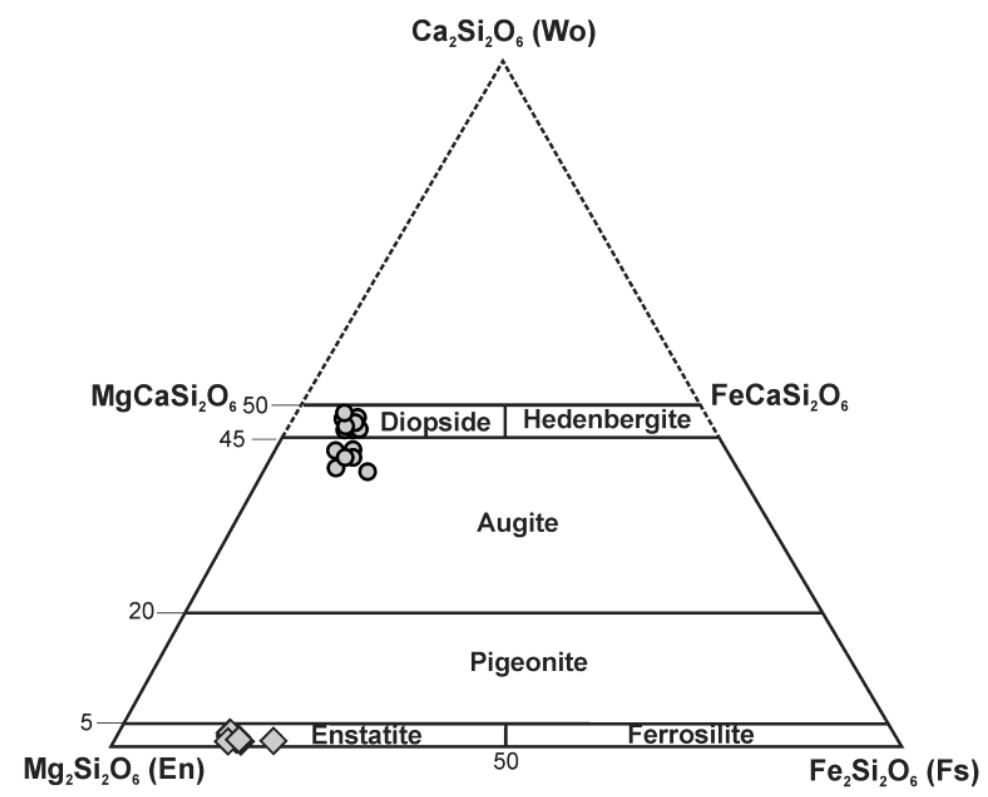

Figure 3. Diagram of classification of pyroxenes [24]

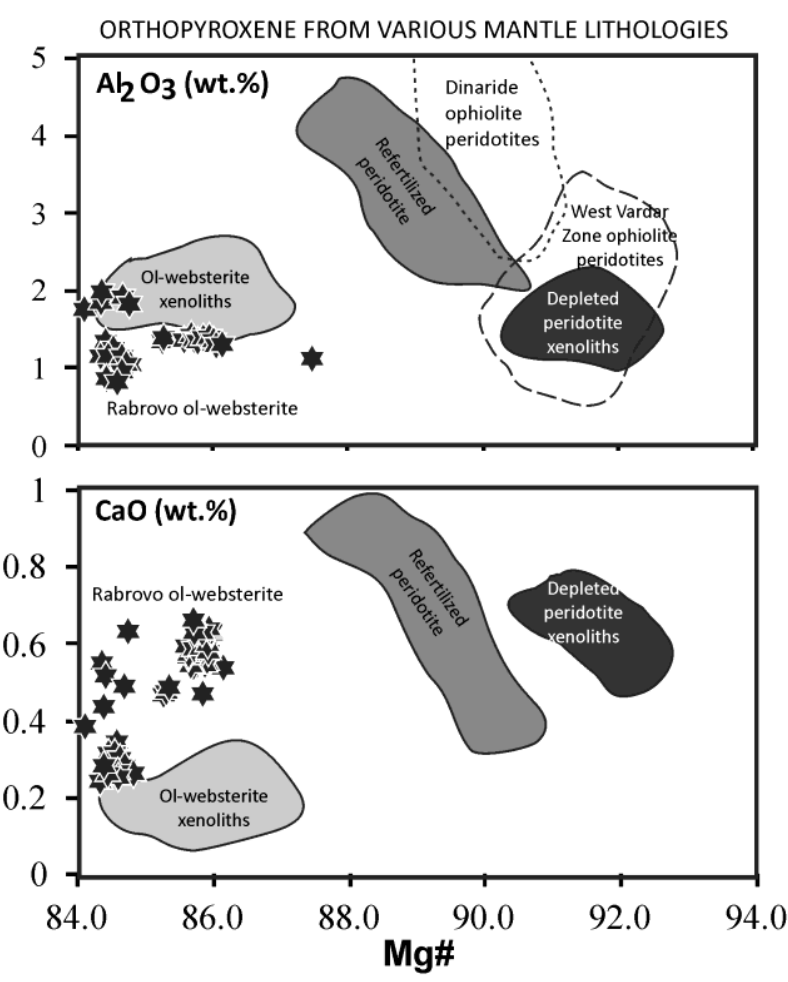

Figure 4. $\mathrm{Mg} \#\left[\mathrm{~mol} \mathrm{MgO} * 100 /\left(\mathrm{MgO}+\mathrm{FeO}^{\mathrm{t}}\right)\right]$ vs $\mathrm{Al}_{2} \mathrm{O}_{3}$ (a) and $\mathrm{CaO}$ (b) plots for orthopyroxene; data for the composition of orthopyroxene from other lithologies are from: $[11,13]$ for orthopyroxene from various East Serbian mantle xenoliths and [25] for orthopyroxene from Dinaride and West Vardar peridotites

\section{Geothermometric calculations}

Temperatures were calculated using the olivine-spinel [28-30], clinopyroxene-only [31] and orthopyroxene-only [32] geothermometers for pressures ranging between 2 and 5 kbars. The results of geothermometric calculations are shown in Table 5. The average calculated temperature according to different geothermometers varies between 750 and $850^{\circ} \mathrm{C}$.

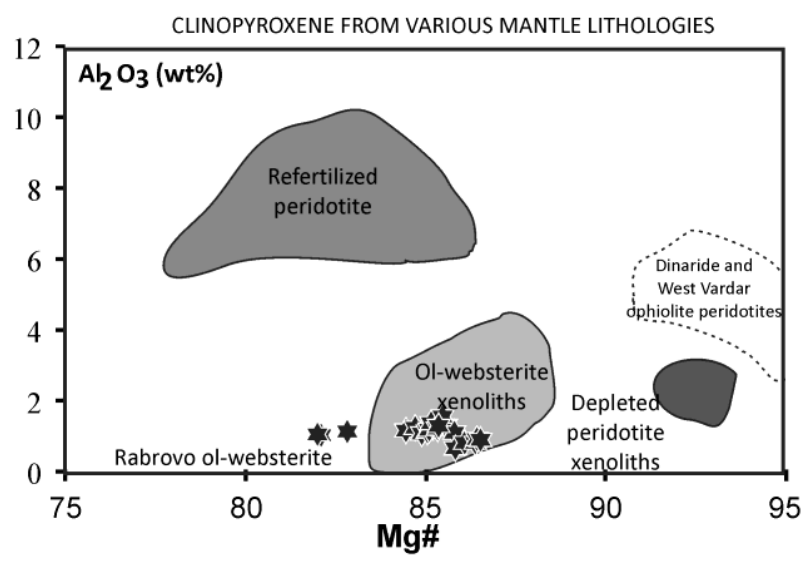

Figure 5. $\mathrm{Mg} \#\left[\mathrm{~mol} \mathrm{MgO} * 100 /\left(\mathrm{MgO}+\mathrm{FeO}^{\mathrm{t}}\right)\right]$ vs $\mathrm{Al}_{2} \mathrm{O}_{3}$ plot for clinopyroxene; data for the composition of clinopyroxene from other lithologies are from: $[11,13]$ for clinopyroxene from various East Serbian mantle xenoliths and [25] for clinopyroxene from Dinaride and West Vardar peridotites

\section{DISCUSSION}

The origin of the olivine websterite veins

The olivine websterite veins found cutting the Rabrovo serpentinite are characterized by remarkably 
fertile compositions. Such compositions fall outside the main compositional trends of common mantle peridotites. These veins are ortho- and clinopyroxene-rich and all their Fe-Mg silicates have relatively low $\mathrm{Mg \#}(<87)$ to be in equilibrium with typical mantle silicates. These characteristics indicate that this lithology cannot be regarded as 'normal' upper mantle. It is generally accepted that such pyroxene-rich rocks are related to magmatic modifications of the upper mantle i.e. that they result from percolations and precipitations of mafic/ultramafic magma and formation of pyroxene-rich domains in the mantle lithosphere [33, 34]. Besides the formation of magmatic mafic and ultramafic veins, this process is also responsible for metasomatic refertilization of previously depleted lithospheric mantle. In such a way, previously variably depleted lherzolite/harzburgite mantle peridotite is transformed into clinopyroxene-rich lherzolite and, in some cases, even wherlite domains [35].

Table 5. Calculated temperatures according to different geothermometers

\begin{tabular}{lll}
\hline $\begin{array}{l}\text { Olivine-spinel geothermometer } \\
\text { [27] Fabriés, 1979 }\end{array}$ & $690-770^{\circ} \mathrm{C}\left(\right.$ average $\left.=732, \mathrm{SEE}=25^{\circ} \mathrm{C}\right)$ & $\mathrm{n}=12$ \\
[28] Roeder et al., 1979 & $660-770^{\circ} \mathrm{C}\left(\right.$ average $\left.=709, \mathrm{SEE}=36^{\circ} \mathrm{C}\right)$ & $\mathrm{n}=12$ \\
[29] Ballhaus et al., 1991 & $630-700^{\circ} \mathrm{C}\left(\right.$ average $\left.=766, \mathrm{SEE}=25^{\circ} \mathrm{C}\right)$ & $\mathrm{n}=12$ \\
Clinopyroxene only geothermometer & & \\
[30] Nimis and Taylor, 2000 & $770-950^{\circ} \mathrm{C}\left(\right.$ average $\left.=845, \mathrm{SEE}=51^{\circ} \mathrm{C}\right)$ & $\mathrm{n}=20$ \\
Orthopyroxene only geothermometer & & \\
[31] Witt-Eickschen and Seck, 1991 & $690-890^{\circ} \mathrm{C}\left(\right.$ average $\left.=772, \mathrm{SEE}=60^{\circ} \mathrm{C}\right)$ & $\mathrm{n}=20$ \\
\hline
\end{tabular}

However, the above mentioned modifications of the normal upper mantle material commonly produces clinopyroxene-rich lithologies and, additionally, such lithologies contain typically Fe-Ti$\mathrm{Al}-\mathrm{Ca}$ rich silicates. By contrast, the Rabrovo olivine websterite possesses a list of other characteristics that are unusual for lithologies having formed by modifications caused by mafic melts. First, the Rabrovo olivine websterite is rich in orthopyroxene (> $50 \%$ vol.) that usually predominates over clinopyroxene. It is generally known that this mineral normally decrease in abundance during mafic metasomatism because this phase is not stable in Si-undersaturated magmas or those close to silica saturation [36].

The presence of orthopyroxene-rich mantle rocks have been reported by many authors [7, 37-39]. The origin and evolution of these lithologies have been variously interpreted. Namely, by: (i) deserpentinization of the normal peridotitic upper mantle (e.g. [40]), (ii) metasomatism induced by percolating Si-rich melts/fluids [7, 37], or (iii) direct crystallization from a silica saturated melt (e.g. [41]). The first two interpretations are not likely scenarios for the origin of melts from which the Rabrovo olivine websterite crystallized. On the contrary, these rocks show many characteristics that can be associated with crystallization of Si-rich lithospheric magmas. This is indicated from textural relationships that are found in the studied olivine websterite, at first place, from the presence of cumulate-like texture (see Figure 2c). Moreover, these rocks lack fibrous orthopyroxene or olivine relicts in orthopyroxene, which are commonly found after deserpentinization and/or fluid-induced metasomatic processes. Mineral chemistry data also support this conclusion. Namely, Mg\# and $\mathrm{NiO}$ contents of the orthopyroxene from the Rabrovo olivine websterite is rather low $(<87$ and $<0.06 \mathrm{wt} . \%$, respectively), to support an origin via deserpentinization and fluidinduced metasomatism. Therefore, it is more logical that the studied olivine websterite veins originated by crystallization of a primarily silica- and $\mathrm{Mg}$-rich, presumably, boninite-like magma. Early crystallization of orthopyroxene, which is suggested by textural relationships, indicates silica-saturated or oversaturated melts, most probably with more than 53 wt.\% $\mathrm{SiO}_{2}$ (e.g. [42]). Higher modal abundance of clinopyroxene in combination with relatively low $\mathrm{Mg \#}$ in olivine and pyroxenes and somewhat higher $\mathrm{CaO}$ contents in orthopyroxene, can suggest that these olivine websterites crystallized from evolved magmas.

\section{Geodynamic implications}

Notwithstanding which interpretation for the origin of the Rabrovo olivine websterite we adopt, it is very likely that the formation of these rocks was associated with a supra-subduction setting. 
Similar orthopyroxene-rich mantle domains are found at many places worldwide: Papua New Guinea [7], USA [38, 43, 44], Kamchatka [40], Canada [45], Andes [46], Philippines [47], Zabargard peridotite, Red Sea [48], and Cabo Ortegal peridotite, Spain [49]. Most these localities have a close spatial and temporal relation to subduction.

It is worth noting that the studied olivine websterite veins are found cutting a serpentinite block from the East Vardar Zone mélange. It is known that the East Vardar ophiolite is dominated by harzburgites and is considered to be the most depleted one in the Balkan Peninsula. As previously mentioned, [10, 11, 13] argued that orthopyroxenerich mantle xenoliths in Serbia also originated as lithospheric precipitates of arc-related magmas. They suggested that these high-Mg and high-Si magmas resulted from melting of a highly refractory harzburgitic source, most probably due to a $\mathrm{H}_{2} \mathrm{O}$ flux. Such melting processes are very common in fore-arc regions. Analogously, the formation of the studied olivine websterite vein could have resulted from melting of highly refractory mantle peridotites in the presence of $\mathrm{H}_{2} \mathrm{O}$ flux and depression of melting temperatures. The orthopyroxene-rich mantle xenoliths differ from the studied olivine websterite by having abundant carbonate and displaying much higher $\mathrm{Cr} \#$ values in their spinels. However, this cannot disturb the postulated petrogenetic analogy of these two lithologies. As shown by Cvetković and coauthors $[10,11,13]$ the carbonate present in the East Serban orthopyroxene-rich mantle xenoliths is unrelated to their original composition but was likely introduced via later metasomatic processes. On the other hand, higher Cr\#s in spinels from the xenoliths can be explained because the source from which this lithology originated was associated with higher degree of depletion.

The fact that there is a contrast in $\mathrm{Fe}-\mathrm{Mg}$ distribution between the silicates of the olivine websterite and normal depleted peridotite suggests that these websterite domains did not reside long in the mantle before they were tectonically emplaced and reworked in the mélange. This is corroborated by a very large range temperature range that is obtained by thermometric calculations, which can suggest fast and incomplete equilibration. This, in turn, indicates that subduction processes that were responsible for subduction, the most likely candidate is the Mesozoic subduction related to closure of the Tethyan Ocean [21].

\section{CONCLUSIONS}

The presence of olivine websterite veins cutting the Rabrovo serpentinite block in the mélange is considered as evidence that the East Vardar Zone ophiolite belt formed in a suprasubduction setting. Textural relationships suggest that the olivine websterite vein represents relicts of magmatic precipitates at crustal or subcrustal (?) depths. The average temperature of equilibration is estimated to $750-850{ }^{\circ} \mathrm{C}$. Low $\mathrm{Mg} \#$ values in silicates of the studied olivine websterite suggests that these magmas did not reside long in the lithosphere. Major element chemical compositions of the main minerals composing the olivine websterite indicate that the studied veins formed by crystallization of silica saturated, $\mathrm{MgO}$-rich primary magmas that commonly originate by melting of highly depleted peridotite in sub-arc settings. This study strongly suggests that the investigation of pyroxene-rich lithospheric rocks can be very useful palaeotectonic indicators, especially recording possible effects of subduction processes.

Acknowledgements: This study is carried out in the frame of bilateral collaboration between the Macedonian Academy of Sciences and Arts and the Serbian Academy of Sciences and Arts.

\section{REFERENCES}

[1] A. H. F. Robertson, S. Karamata, The role of subduction-accretion processes in the tectonic evolution of the Mesozoic Tethys in Serbia. Tectonophysics 234 (1994) pp. 73-94.

[2] M. S. Schmid, D. Bernoulli, B. Fügenschuh, L. Matenco, S. Schefer, R. Schuster, M. Tischler, K. Ustaszewski, The Alps-Carpathians-Dinaridesconnection: A correlation of tectonic units. Swiss J. Geosci. 101 (2008) pp. 139-183.

[3] Z. Maksimović, V. Majer,. Accessory spinels of two main zones of alpine ultramafic rocks in Yugoslavia. Bulletin - Academie Serbe des Sciences et des Arts 21 (1981) 47-58.

[4] B. Lugović, R. Altherr, I. Raczek, A.W. Hofmann, V. Majer, Geochemistry of peridotites and mafic igneous rocks from the Centarl Dinaric Ophiolite Belt, Yugoslavia. Contrib. Miner. Petrol. 106 (1991) pp. 201-216.

[5] B. A. Bazylev, A. Popevic, S. Karamata, N. N. Kononkova, S.G. Simakin, J. Olujic, L. Vujnovic, E. Memovic, Mantle peridotites from the Dinaridic ophiolite belt and the Vardar zone western belt, central Balkan: A petrological comparison. Lithos 108 (2009) pp. 37-71. 
[6] A. Popević, S.P. Korikovsky, S. Karamata, Garnet clinopyroxenite from Bistrica, southern Zlatibor, Serbia. Bulletin of the Geological Society of Greece XXVIII, 2 (1993) pp. 93-103.

[7] B. I. A. McInnes, M. Gregoire, R. A. Binns, P. M. Herzig, M. D. Hannington, Hydrous metasomatism of oceanic sub-arc mantle, Lihir Papua New Guinea: petrology and geochemistry of fluid-metasomatised mantle wedge xenoliths. Earth Planet. Sci. Lett. 188 (1-2) (2001) pp. 169-183.

[8] Z. Maksimović, L. Jovanović, Geochemical processes in upper mantle in reference to spinel peridotites in the Balkan Peninsula. 27 th International Geological Congress, Moskva, 1984, 5, p. 342.

[9] M. Božović, D. Prelević, R. L. Romer, M. Barth, P. Den Bogaard, B. Boev, The Demir Kapija Ophiolite, Macedonia (FYROM): a Snapshot of Subduction Initiation within a Back-arc. J. of Petr., 54, 7 (2013) pp. 1427-1453.

[10] V. Cvetkovic, H. Downes, D. Prelevic, M. Jovanovic, M. M. Lazarov, Characteristics of the lithospheric mantle beneath East Serbia inferred from ultramafic xenoliths in Palaeogene basanites. Contrib. Mineral. Petrol. 148 (2004) pp. 335-357.

[11] V. Cvetkovic, M. Lazarov, H. Downes, D. Prelevic, Modification of the subcontinental mantle beneath East Serbia: Evidence from orthopyroxenerich xenoliths. Lithos 94 (2007a) pp. 90-110.

[12] D. Prelević, C. Akal, S. F. Foley, Orogenic vs anorogenic lamproites in a single volcanic province: Mediterranean-type lamproites from Turkey IOP Conference Series: Earth and Env. Sci. 2 (2008) p. 012024.

[13] V. Cvetković, H. Downes, D. Prelević, M. Lazarov, K. Resimić-Šarić, Geodynamic significance of ultramafic xenoliths from Eastern Serbia: Relics of sub-arc oceanic mantle? Journal of Geodynamics 43 (2007b) pp. 504-527.

[14] J. Pamic, B. Tomljenovic, D. Balen, Geodynamic and petrogenetic evolution of Alpine ophiolites from the central and NW Dinarides: an overview. Lithos 65 (2002) pp. 113-142.

[15] V. Bortolotti, G. Principi, Tethyan ophiolites and Pangea break-up. Island Arc 14 (2005) pp. 442-470.

[16] M. Zelic, M. Marroni, L. Pandolfi, B. Trivic, Tectonic setting of the Vardar suture zone (Dinaric-Hellenic belt): The example of the Kopaonik area (Southern Serbia). Ofioliti 35 (2010) pp. 49-69.

[17] M. Marovic, I. Djokovic, L. Pesic, M. Toljic, N. Gerzina, The genesis and geodynamics of Cenozoic sedimentation provinces of the central Balkan Peninsula. Geotectonics 34 (2000) 415-427.

[18] S. Lepitkova, Petroloski, geohemijski i izotopski proucuvanja na peridotitite od vnatresniot dinarski ofiolitski pojas vo Republika Makedonija. PhD
Thesis, Ss. Cyril and Methodius University Skopje, (2002) 333 pp.(in Macedonian)

[19] B. Anders, T. Reischmann, D. Kostopolous, U. Poller, The oldest rocks of Greece: first evidence for a Precambrian terrane within the Pelagonian Zone. Geol. Mag. 143 (2006) pp. 41-58.

[20] S. Karamata, The geological framework of the Balkan Peninsula: its origin due to the approach, collision and compression of Gondwanian and Eurasian units, in: Tectonic Development of the Eastern Mediterranean Region, A.H.F. Robertson, D. Mountrakis (Eds), Geol. Soc. Lond. Spec. Publ., Geological Society, London, Special Publications 260 (2006) pp. 155-178.

[21] A. H. F. Robertson, S. Karamata, K. Šarić, Overview of ophiolites and related units in the Late Palaeozoic-Early Cenozoic magmatic and tectonic development of Tethys in the northern part of the Balkan region. Lithos, 108 (2009) pp. 1-36.

[22] J. T. Armstrong, A package of correction programs for the quantitative electron microbeam analysis of thick polished sections, thin films and particles. Microbeam Analysis 4 (1995) 177-200.

[23] T. Ivanovski, Tolkuvač za listot Gevgelija, OGK 1:100.000. Sojuzen geološki zavod Beograd, (1970) $52 \mathrm{pp}$.

[24] N. Morimoto, J. Fabries, A.K. Ferguson, I.V. Ginzburg, M. Ross, F.A. Seifert, J. Zussman, Nomenclature of pyroxenes. Am. Mineral. 62 (1988) pp. 53-62.

[25] D. Prelević, G. Brügmann, M. Božović, S.F. Foley, V. Cvetković, Z. Maksimović, Os-isotopes constraints on the dynamics of orogenic mantle: the case of Central Balkans. Gondwana Research (2013) Accepted.

[26] M. Božović, Petrology and Geochemistry of the Balkan ophiolites. Johannes Gutenberg University Mainz, PhD Thesis, (2013) 254 pp.

[27] S. Arai, Characterization of spinel peridotites by olivine-spinel compositional relationships: review and interpretation. Chem. Geol. 111 (1994), pp. 191-204.

[28] J. Fabries, Spinel-olivine geothermometry in peridotites from ultramafic complexes. Contrib. Mineral. Petrol. 69 (1979) 329-336.

[29] P. L. Roeder, J. H. Campbell, H. E. Jamieson, A reevaluation of the olivine-spinel geothermometer. Contrib. Mineral. Petrol. 68 (1979) 325334.

[30] C. Ballhaus, R. F. Berry, D. H. Green, High pressure experimental calibration of the olivine orthopyroxene-spinel oxygen geobarometer: implications for the oxidation state of the upper mantle. Contrib. Mineral. Petrol. 107 (1991) 27-40.

[31] P. Nimis, W. R. Taylor, Single clinopyroxene thermobarometry for garnet peridotites. Calibration and testing of a Cr-in-cpx barometer and 
an enstatite-in-cpx thermometer. Contrib. Mineral. Petrol. 139 (2000) 541-554.

[32] G. Witt-Eickschen, H.A. Seck, Solubility of Ca and $\mathrm{Al}$ in orthopyroxene from spinel peridotite: an improved version of an empirical geothermometer. Contrib. Mineral. Petrol. 106 (1991) 431-439.

[33] A. V. McGuire, S. B. Mukasa, Magmatic modification of the uppermost mantle beneath the Basin and Range to Colorado Plateau transition zone: evidence from xenoliths, Wikieup, Arizona. Contrib. Mineral. Petrol. 128 (1997) pp. 52-65.

[34] W. L. Griffin, S. R. Shee, C. G. Ryan, T. T. Win, B. A. Wyatt, Harzburgite to lherzolite and back again: metasomatic processes in ultramafic xenoliths from the Wesselton Kimberlite, Kimberley, South Africa. Contrib. Mineral. Petrol. 134 (1999) pp. 232-250.

[35] R. L. Carpenter, A. D. Edgar, Y. Thibault, Origin of spongy textures in clinopyroxene and spinel from mantle xenoliths, Hessian Depression, Germany. Mineral. and Petrol., 74 (2002) 149162.

[36] M. S. Ghiorso, I.S.E. Carmichael, Modeling magmatic systems: petrologic applications. Reviews in Mineralogy, 17 (1987) pp. 467-499.

[37] B. I. A. McInnes, M. Cameron Eion, Carbonated, alkaline hybridizing melts from a sub-arc environment: Mantle wedge samples from the Tabar-Lihir-Tanga-Feni arc, Papua New Guinea. Earth Planet.Sci. Lett. 122 (1994) pp. 125-141.

[38] I. E. Ertan, W.P. Leeman, Metasomatism of Cascades subarc mantle: evidence from a rare phlogopite orthopyroxenite xenolith. Geology 24 (1996) pp. 451-454.

[39] I. Chane-Fo, M. Godard, A. Tommasi, A. Vauchez, F. Belley, H. Lapierre, Y. Tardy, Partial melting and melt circulation above a subduction zone: a petrophysical and geochemical study of the refractory peridotites and pyroxenites from Murray Ridge, British Columbia. Geophys. Res. Abstrs. 5, 2003, p. 11289.

[40] S. Arai, S. Ishimaru, V. Okrugin, Metasomatized harzburgite xenoliths from Avacha volcano as fragments of mantle wedge of the Kamchatka arc: implication for the metasomatic agent. Island Arc 12 (2003) pp. 233-246.

[41] A. Al-Boghdady, M. Economou-Eliopoulos, Fluid inclusions in chromite from a pyroxenite dyke of the Pindos ophiolite complex. Chemie der Erde Geochemistry 65 (2005) pp. 191-202.

[42] S. A. Morse, Kiglapait mineralogy, II: Fe-Ti oxide minerals and the activities of oxygen and silica. J.Petrol. 21 (1980) 685-719.

[43] D. Smith, J. C. A. Riter, Genesis and evolution of low-Al orthopyroxene in spinel peridotite xenoliths, Grand Canyon Field, Arizona. Contrib. Mineral. Petr. 127 (1997) pp. 391-404.

[44] D. Smith, J. C. A. Riter, S. A. Mertzman, Waterrock interactions, orthopyroxene growth, and $\mathrm{Si}$ enrichment in the mantle: evidence in xenoliths from the Colorado Plateau, southwestern United States. Earth Planet. Sci. Lett. 165 (1999) 45-54.

[45] I. Chane-Fo, M. Godard, A. Tommasi, A. Vauchez, F. Belley, H. Lapierre, Y. Tardy, Partial melting and melt circulation above a subduction zone: a petrophysical and geochemical study of the refractory peridotites and pyroxenites from Murray Ridge, British Columbia. Geophys. Res. Abstrs. 5 (2003) p.11289.

[46] R. Kilian, C. Franzen, M. Koch, B. Stern, C.R. Altherr, Metasomatism of the mantle wedge below the southern Andes. International Symposium on Andean Geodynamics, Oct. 1999, Göttingen, Abstract Volume.

[47] S. Arai, M. Kida, Origin of fine-grained peridotite xenoliths from Iraya volcano of Batan Island, Philippines: deserpentinization or metasomatism at the wedge mantle beneath an incipient arc? Island Arc 9 (2000) pp. 458-471.

[48] G. Schmidt, H. Palme, K. L. Kratz, G. Kurat, Are highly siderophile elements (PGE, Re and Au) fractionated in the upper mantle of the earth? New results on peridotites from Zabargad. Chem. Geol. 163 (2000) pp. 167-188.

[49] J. F. Santos, U. Schaerer, J. I. Gil Ibarguchi, J. Girardeau, Genesis of pyroxenite-rich peridotite at Cabo Ortegal (NW Spain); geochemical and $\mathrm{Pb}-\mathrm{Sr}-$ $\mathrm{Nd}$ isotope data. J. Petrol 43 (2002) pp. 17-43. 


\title{
ОЛИВИНСКИ ВЕБСТЕРИТСКИ ЖИЦИ КОИ ГО СЕЧАТ СЕРПЕНТИНСКИОТ МАСИВ НА РАБРОВО (ЈУЖНА МАКЕДОНИЈА) \\ НОВИ СОГЛЕДУВАЊА ЗА ЛАЧНАТА СТРУКТУРА ВО ИСТОЧНИОТ ОФИОЛИТСКИ ПОЈАС НА ВАРДАРСКАТА ЗОНА
}

\author{
Блажо Боев ${ }^{1}$, Дејан Прелевиќ ${ }^{2}$ Милица Божовиќ ${ }^{2}$, Сузана Ериќ ${ }^{3}$, Владица Цветковиќ \\ ${ }^{1}$ Универзитет „Гоце Делчев““, Штип, Република Македонија \\ ${ }^{2}$ Johannes Gutenberg-Universität, Institut für Geowissenschaften, fb 09, Mineralogie, Mainz, Germany \\ ${ }^{3}$ University of Belgrade - Faculty of Mining and Geology, Belgrade, Serbia
}

\begin{abstract}
Во трудот е прикажана петрографијата и хемијата на минералите од ултрабазичните жилни карпи кои ги сечат серпентинитите на Раброво (во близината на Валандово, југоисточна Македонија). Серпентинитите се појавуваат во вид на блокови во раседните зони од тектонскиот меланж во источната Вардарска субзона.Жилните карпи одговараат на средно зрнести оливински вебстерити и понекогаш покажуваат кумулатни текстури. Изградени се од ортопироксен, клинопироксен оливин и спинел. Составот на пироксенот укажува на тоа дека тие не се репрезент на нормална литосверска обвивка. На основа на главните карактеристики на офиолитите од источната вардарска субзона може да се констатира дека тие представуваат типични супра субдукциски офиолити.
\end{abstract}

Клучни зборови: ултрабазични карпи; ортопироксени; субдукција; Балкански Полуостров 